\title{
TWO THEOREMS ON GALOIS COHOMOLOGY ${ }^{1}$
}

\author{
MICHAEL ROSEN
}

1. Introduction. Let $k$ and $K$ be algebraic number fields, $K$ a finite extension of $k$ with Galois group $G$. H. Yokoi has considered the ring of integers $O_{K}$ in $K$ as a $Z[G]$ module (see [2]). In particular, he has proven

THEOREM. If both $K$ and $k$ are Galois over the rationals $Q$, and $G$ is cyclic of prime order, then $H^{m}\left(G, O_{K}\right) \approx H^{n}\left(G, O_{K}\right)$ for all integers $m$ and $n$.

We will prove a generalization of this. Namely

THEOREM 1. If $G$ is a cyclic group, then ord $H^{m}\left(G, O_{K}\right)=\operatorname{ord} H^{n}\left(G, O_{K}\right)$ for all integers $m$ and $n$.

Notice that we have dropped the hypothesis that both $k$ and $K$ be Galois over the rationals.

To see how Theorem 1 generalizes Yokoi's result, remember that if $G$ has prime order $p$, then multiplication by $p$ annihilates all the cohomology groups. Thus in this case the cohomology groups are determined up to isomorphism by their order.

The technique used to prove Theorem 1 can be used to prove other results of a similar nature. In the same situation as above let us consider $U_{K}$, the units of $K$, as a $Z[G]$ module. Then we have

THEOREM 2. Let $G$ be a cyclic group, and suppose that no infinite prime of $k$ is ramified in $K$. If ord $G=n$, then $n$ ord $H^{2 r}\left(G, U_{K}\right)$ $=$ ord $H^{2 s+1}\left(G, U_{K}\right)$ for all integers $r$ and $s$.

The hypothesis about no infinite prime ramifying is satisfied, for example, when $K$ is totally real or when $n$ is odd.

2. Proofs of the theorems. The proofs of both theorems are easy consequences of the following lemma which is a direct generalization of a result of Chevalley in Herbrand quotients (see [1]). It has come to my attention that this generalization has been discovered independently by Dr. J. Smith of Michigan University.

We need some notation. From now on $G$ will be a cyclic group of order $n, \sigma$ a generator of $G$, and when $d \mid n, G(d)$ will be the unique

Received by the editors October 18, 1965 and, in revised form, March 24, 1966.

1 This paper was written when the author held an O.N.R. Research Associateship (O.N.R. 432). 
subgroup of $G$ having order $d . K(d)$ will be the cyclotomic field of $d$ th roots of unity, $O(d)$ the ring of integers in $K(d)$, and $\zeta(d)$ a primitive $d$ th root of unity.

When $d$ is a prime power, $p^{i},(1-\zeta(d))$ is a prime ideal in $O(d)$, whose residue class field has $p$ elements. On the other hand, when $d$ is composite $1-\zeta(d)$ is a unit. This is seen as follows. Let $d=p_{1} p_{2} q$, where $p_{1}$ and $p_{2}$ are distinct primes. Notice that $\zeta(d)^{p_{1} q}=\zeta\left(p_{2}\right)$ and $\zeta(d)^{p_{2} q}=\zeta\left(p_{1}\right)$. This shows that $1-\zeta(d)$ divides both $1-\zeta\left(p_{1}\right)$ and $1-\zeta\left(p_{2}\right)$. Taking absolute norms, we see that the norm of $1-\zeta(d)$ divides both $p_{1}$ and $p_{2}$. Thus the norm of $1-\zeta(d)$ is a unit, and consequently $1-\zeta(d)$ is a unit.

Let $A$ be a finitely generated $Z[G]$ module. The Herbrand quotient, $q(A)$, is defined to be the ratio of ord $H^{0}(G, A)$ to ord $H^{1}(G, A)$.

Let $A^{G(d)}$ be the subset of $A$ left fixed by $G(d)$, and define $r(d)$ to be the $Z$ rank of $A^{G(d)}$.

Lemma. Let $n=\prod_{p} p^{t(p)}$ be the prime decomposition of $n$. Then $q(A)=\prod_{p \mid n} p^{8(p)}$ where

$$
s(p)=t(p) r(n)-\sum_{i=1}^{t(p)} \phi\left(p^{i}\right)^{-1}\left(r\left(n / p^{i}\right)-r\left(n / p^{i-1}\right)\right) .
$$

Proof. Notice to begin with that $Q[G] \approx Q[x] /\left(x^{n}-1\right)$ where $x$ is an indeterminate. We have $x^{n}-1=\prod_{d \mid n} \Phi_{d}(x)$ where $\Phi_{d}(x)$ is the cyclotomic polynomial of $d$ th roots of unity. Consequently, $Q[G]$ $\approx \sum_{d \mid n} K(d)$. Each $K(d)$ becomes an irreducible $Q[G]$ module, where $\sigma$ acts as multiplication by $\zeta(d)$.

Consider $V=Q \otimes A . V$ is a $Q[G]$ module. Thus $V \approx \sum_{d \mid n} a(d) K(d)$ where the $a(d)$ are certain nonnegative integers. We easily deduce the existence of a $Z[G]$ submodule $B$ of $A$ such that $A / B$ is finite, and $B \approx \sum_{d \mid n} a(d) O(d)$. From the well known properties of the Herbrand quotient we have

$$
q(A)=q(B)=\prod_{d \mid n} q(O(d))^{a(d)} .
$$

We now compute $q(O(d))$. For $d=1, O(d)=Z$ acted on trivially by $G$. Let $N=\sum_{i=0}^{n-1} \sigma^{i}$. Then $H^{0}(G, Z)=Z / N Z=Z / n Z$. Since $G$ is cyclic $H^{1}(G, Z) \approx H^{-1}(G, Z)=Z_{N} /(1-\sigma) Z=(o)$. Thus $q(O(1))=n$.

For $d \neq 1$ we have $O(d)^{G}=(o)$ since $\sigma$ acts as multiplication by $\zeta(d)$. Therefore ord $H^{0}(G, O(d))=1$. On the other hand, $O(d)_{N}$ $=\{a \in O(d) \mid N a=o\}=O(d)$, and $(1-\sigma) O(d)=(1-\zeta(d))$. Thus $H^{1}(G, O(d)) \approx H^{-1}(G, O(d))=O(d) /(1-\zeta(d))$. The remarks preceding this lemma now show that $q(O(d))=1$ if $d$ is composite and $q(O(d))$ $=p^{-1}$ if $d=p^{i}$ is a prime power. 
Putting together the information we now have, we get that $q(A)$ $=\prod_{p \mid n} p^{s(p)}$ where

$$
s(p)=t(p) a(1)-\sum_{i=1}^{t(p)} a\left(p^{i}\right)
$$

To relate the $a(d)$ with the $r(d)$ notice that the $Z$ rank of $A^{G(d)}$ is equal to the $Q$ dimension of $V^{G(d)}$. The group $G\left(n / p^{i}\right)$ is generated by $\sigma^{p^{i}}$. From the way that $\sigma$ acts it follows that $V^{G\left(n / p^{i}\right)}$ $=\sum_{j=0}^{i} a\left(p^{j}\right) K\left(p^{j}\right)$. Therefore, $r\left(n / p^{i}\right)=\sum_{j=0}^{i} \phi\left(p^{j}\right) a\left(p^{j}\right)$. Solving for $a\left(p^{i}\right)$ we get that $a\left(p^{i}\right)=\phi\left(p^{i}\right)^{-1}\left(r\left(n / p^{i}\right)-r\left(n / p^{i-1}\right)\right)$. This completes the proof.

Proof of Theorem 1. Since $G$ is cyclic the cohomology groups are periodic of order 2. It is thus sufficient to show that $q\left(O_{K}\right)=1$. If $[K: Q]=N$, then the $Z$ rank of $O_{K}^{G(d)}=N / d$. Substituting this information into the formula of the lemma we see that, indeed, $q\left(O_{K}\right)$ $=1$.

COROLlaRy. If $G$ is cyclic of square free order then $H^{n}\left(G, O_{K}\right)$ $\approx H^{m}\left(G, O_{K}\right)$ for all integers $m$ and $n$.

Proof. The restriction map gives a monomorphism of the $p$ primary component of $H^{i}\left(G, O_{K}\right)$ into $H^{i}\left(G(p), O_{K}\right)$. It follows that the $p$-primary components of the cohomology groups under consideration are elementary. These groups are thus determined up to isomorphism by their order.

Proof of Theorem 2. Let $K$ be an algebraic number field. Denote by $r_{1}(K)$ the number of real primes of $K$, and by $r_{2}(K)$ one half the number of complex primes. The Dirichlet Unit Theorem states that rank $\left(U_{K}\right)=r_{1}(K)+r_{2}(K)-1$. If $k$ is a subfield of $K$, the condition that no infinite prime ramify in $K$ means that the extension of every real place is real. This implies rank $\left(U_{K}\right)=[K: k] \operatorname{rank}\left(U_{k}\right)+[K: k]$ -1 . Using the notation of the lemma, with $A=U_{K}$, we have $r(1)$ $=d r(d)+d-1$. Substituting this into the formula of the lemma we get $s(p)=-t(p)$ and thus $q\left(U_{K}\right)=n^{-1}$. This finishes the proof.

\section{BiBLIOGRAPHY}

1. C. Chevalley, Class field theory, Nagoya University, Japan, 1953-1954.

2. H. Yokoi, On the Galois cohomology group of the ring of integers in an algebraic number field, Acta Arith. 8 (1962/1963), 243-250.

BRANDEIS UNIVERSITY 